This item was submitted to Loughborough's Research Repository by the author.

Items in Figshare are protected by copyright, with all rights reserved, unless otherwise indicated.

\title{
Predicting maximum eccentric strength from surface EMG measurements
}

PLEASE CITE THE PUBLISHED VERSION

PUBLISHER

(C) Elsevier

VERSION

AM (Accepted Manuscript)

LICENCE

CC BY-NC-ND 4.0

REPOSITORY RECORD

Pain, Matthew T.G., and Stephanie E. Forrester. 2019. "Predicting Maximum Eccentric Strength from Surface EMG Measurements". figshare. https://hdl.handle.net/2134/6536. 
This item was submitted to Loughborough's Institutional Repository (https://dspace.lboro.ac.uk/) by the author and is made available under the following Creative Commons Licence conditions.

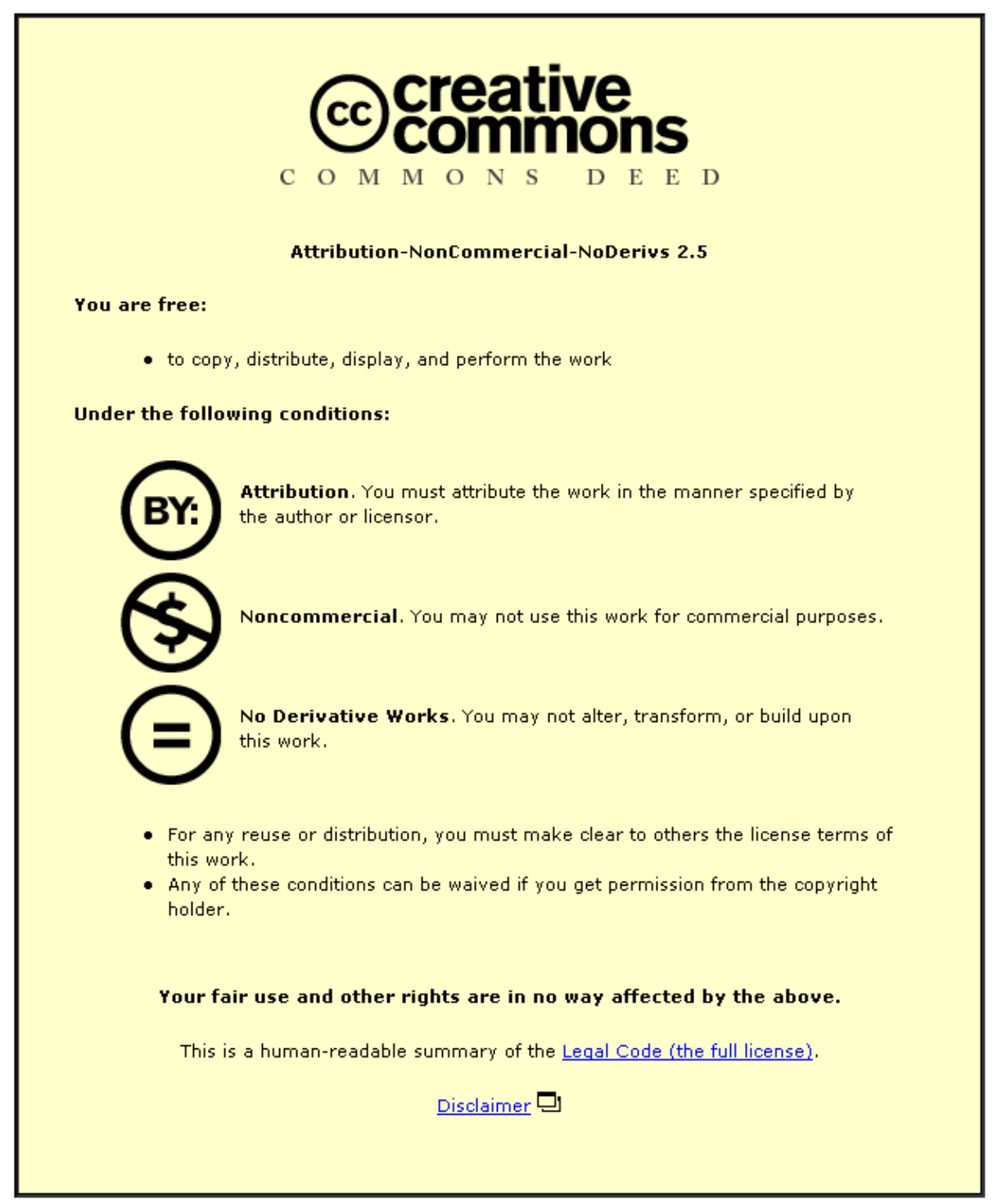

For the full text of this licence, please go to: http://creativecommons.org/licenses/by-nc-nd/2.5/ 


\title{
Predicting maximum eccentric strength from surface EMG measurements
}

\author{
${ }^{1}$ Matthew T.G. Pain and ${ }^{2}$ Stephanie E. Forrester \\ ${ }^{1}$ School of Sport and Exercise Sciences, Loughborough University, UK \\ ${ }^{2}$ Wolfson School of Mechanical and Manufacturing Engineering, Loughborough University, UK
}

\begin{abstract}
The origin of the well documented discrepancy between maximum voluntary and in vitro tetanic eccentric strength has yet to be fully understood. This study aimed to determine whether surface EMG measurements can be used to reproduce the in vitro tetanic force - velocity relationship from maximum voluntary contractions. Five subjects performed maximal knee extensions over a range of eccentric and concentric velocities on an isovelocity dynamometer whilst EMG from the quadriceps were recorded. Maximum voluntary (MVC) force - length - velocity data were estimated from the dynamometer measurements and a muscle model. Normalised amplitude length - velocity data were obtained from the EMG signals. Dividing the MVC forces by the normalised amplitudes generated EMG corrected force - length - velocity data. The goodness of fit of the in vitro tetanic force - velocity function to the MVC and EMG corrected forces was assessed. Based on a number of comparative scores the in vitro tetanic force - velocity function provided a significantly better fit to the EMG corrected forces compared to the MVC forces $(\mathrm{p} \leq 0.05)$, Furthermore, the EMG corrected forces generated realistic in vitro tetanic force velocity profiles. A $58 \pm 19 \%$ increase in maximum eccentric strength is theoretically achievable through eliminating neural factors. In conclusion, EMG amplitude can be used to estimate in vitro tetanic forces from maximal in vivo force measurements, supporting neural factors as the major contributor to the difference between in vitro and in vivo maximal force.
\end{abstract}

Keywords: tetanic; force - velocity; neural; quadriceps; voluntary contraction

\section{INTRODUCTION}

Maximum strength and power varies with the velocity of muscular contractions and the length of the muscle fibres. The tetanic force - velocity relationship in isolated muscle fibres follows a well established profile characterised by an eccentric force plateau at approximately 1.5 - 1.9 times the isometric value, and a hyperbolic decay in force with increasing shortening velocity (Hill, 1938; Edman et al., 1978; Harry et al., 1990). In vivo measurements of maximum voluntary (MVC) force - velocity show differences to the in vitro tetanic profile, with eccentric forces not increasing much above isometric and tending to decline with increasing lengthening velocity (Dudley et al., 1990, Westing et al., 1990). Consequently, maximum voluntary eccentric strength is approximately $50 \%$ lower than the value one might expect based on maximum isometric measurements and in vitro tetanic force - velocity behaviour.

Using transcutaneous electrical stimulation to supplement maximum voluntary contractions increases eccentric knee extension torque to above MVC, but has no significant effect on concentric torque (Dudley et al., 1990; Westing et al., 1990). Using transcutaneous electrical stimulation as the sole source of knee extensor activation results in subjects not being able to tolerate the development of maximal torques. Constant stimulation levels that produce $40-$ $60 \%$ of MVC were used by Dudley et al. (1990) to reproduce a torque - velocity profile that 
was similar to the in vitro tetanic profile The maximum eccentric torque was 1.4 times the isometric value and did not drop off at higher eccentric velocities.

Surface EMG amplitudes of the vastii and rectus femoris during MVC knee extensions are 10 $-30 \%$ lower for eccentric compared to concentric velocities, which is suggested to represent reduced neural activity during lengthening contractions (Westing et al., 1991; Seger \& Thorstensson, 1994; Beltman et al., 2004). Based on these observations, it is widely believed that the MVC force - velocity profile is modulated by an involuntary changes in neural factors that limit the recruitment and / or discharge rate of motor units (e.g. Aagaard et al., 2000). However, it is uncertain whether neural factors are solely responsible for the difference between MVC and in vitro tetanic eccentric forces. There may be other structural components of whole in vivo muscle tendon complexes, such as changes in pennation angle (Rutherford \& Jones, 1992; Herbert \& Gandevia, 1995; Aagaard et al., 2001) or myofacial force transmission (Rijkelijkhuizen et al., 2005), that contribute.

The marked difference between MVC and in vitro tetanic force levels for eccentric contractions clearly represents an opportunity for improving athletic performance. Given the likely contribution of neural factors (either as a reduction in neural drive or increased neural inhibition), the ability to measure this quantity is highly relevant and gives rise to the question: can the in vitro tetanic force - velocity curve be reproduced from MVC when EMG values are used to correct for involuntary sub-maximal activations?

This study aims to determine whether synchronous measurements of surface EMG, torque, angle and angular velocity for MVC eccentric and concentric knee extensions can be used to reproduce the classical in vitro tetanic force - velocity relationship. The muscle force, length and velocity can be estimated using a muscle model and the corresponding EMG amplitude determined using a continuous wavelet time - frequency analysis (Karlsson et al., 2000). This gives MVC force and EMG amplitude throughout the length - velocity phase space of the task. By mapping globally normalised EMG amplitude onto MVC force, EMG corrected force can be obtained which represents the force that would have been achieved with maximal amplitude at any fibre length and velocity combination. This final stage assumes a linear relationship between EMG amplitude and force over the measurement range, which is task and methodology dependent, therefore some means of validating the EMG corrected forces is required. This is achieved by comparing the MVC and the EMG corrected forces to the theoretical in vitro tetanic force - velocity profile. If the EMG corrected forces provides a better and realistic approximation to the in vitro tetanic force - velocity relationship, then it is likely that changes in neural factors are the major contributor to the difference between in vitro tetanic and MVC forces. This would be unlikely to occur unless the assumptions of a linear EMG amplitude - force relationship was partly valid, or there were specific finely balanced compensating errors.

\section{METHODS}

\section{Subjects}

Five male athletes of national or international standard in power-based sports participated in the study (age $30 \pm 4$ year, body mass $83.5 \pm 13.7 \mathrm{~kg}$, height $1.79 \pm 0.06 \mathrm{~m}$; mean $\pm \mathrm{SD}$ ). The subjects gave written informed consent and the study was conducted in accordance with the approval given by Loughborough University Ethical Advisory Committee.

\section{Experimental protocol}

Maximal effort knee extensions were conducted on an isovelocity dynamometer (Cybex NORM, CSMI, Stoughton, MA, USA) with built-in gravitational torque correction. The 
subjects were seated upright and strapped in to isolate the knee extensors. The dominant leg was strapped tightly to the dynamometer crank arm directly above the ankle joint using a plastic shin guard for protection. Care was taken in setting up the subjects to ensure that the rotational axis of the crank arm was aligned with the centre of the knee joint during near maximal extensions, and crank range of motion was set to the maximum comfortable for the individual. In the isometric trials knee angle was measured using a mechanical goniometer and this data was used to convert the measurements to a joint basis.

\section{Data collection}

After familiarisation and warm up the main protocol, following Yeadon et al. (2006), comprised maximal isometric contractions at crank angles of $15^{\circ}$ through to $75^{\circ}$ in $15^{\circ}$ steps (where $0^{\circ}$ corresponds to anatomical zero), followed by maximal eccentric - concentric contractions at preset crank velocities starting at $50^{\circ} \cdot \mathrm{s}^{-1}$ and increasing in steps of $50^{\circ} \cdot \mathrm{s}^{-1}$ up to a maximum of $300^{\circ} \cdot \mathrm{s}^{-1}$. The latter was designed to provide the preactivation necessary to ensure maximum effort. The trials involved between one (low velocities) and five (high velocities) eccentric - concentric repetitions and a rest interval of at least two minutes between trials was enforced. The protocol finished with the repeat of an isometric trial, where maximum torque was originally recorded, and a dynamic trial $\left(100^{\circ} \cdot \mathrm{s}^{-1}\right)$ to provide a measure of reliability. EMG of the rectus femoris (RF), vastus medialis (VM), vastus lateralis (VL) and biceps femoris (HAM) were recorded using an active bipolar surface electrode system (Biovision, Wehrheim, Germany).

\section{Data processing}

The dynamometer and EMG signals were synchronously sampled at $2000 \mathrm{~Hz}$ and stored for off-line analysis in Matlab (The MathWorks Inc., Natick, MA, USA). The dynamometer signals were low-pass filtered at $8 \mathrm{~Hz}$ using a fourth-order zero-lag Butterworth filter and converted to a joint basis. All data from the joint iso-velocity range were used to provided torque data at a range of angles for each velocity. The joint data were input to a Hill-type muscle model to give the corresponding fibre force, length, and velocity for each of the knee extensors (Appendix A). This resulted in a series of subject specific force - length data for each muscle and velocity that were processed as described below to give muscle specific 2D force - length - velocity surfaces.

For each isometric trial the maximum force was identified. For each dynamic trial the single eccentric and concentric contractions with highest isovelocity forces were identified. The highlighted data from all trials were combined to generate the MVC force - length - velocity surface (Fig. 1A). The EMG signals were bandpass filtered at $10-500 \mathrm{~Hz}$ using a fourthorder zero-lag Butterworth filter. The amplitude of the signals was estimated using a time frequency analysis (Karlsson et al., 2000; von Tascharner, 2000). A complex Morlet wavelet with the wavelet parameters selected to maximize the resolution during dynamic knee extensions was used (Forrester \& Pain, 2007). This allowed the root mean square (RMS) amplitude averaged over $100 \mathrm{~ms}$ windows to be determined. For each isometric trial EMG amplitude corresponding to the previously identified maximum force was determined. For each dynamic trial EMG amplitude corresponding to the previously identified maximal eccentric and concentric contractions was determined. These data from all trials were combined and globally normalised to give an amplitude - length - velocity surface corresponding to the MVC force surface described above (Fig. 1B). Finally, an EMG corrected force - length - velocity surface was determined by dividing the MVC forces by the corresponding normalised EMG amplitudes (Fig. 1C): 


$$
F_{E M G, i}\left(L_{i}, V_{i}\right) \cong \frac{F_{M V C, i}\left(L_{i}, V_{i}\right)}{\operatorname{AMPN}_{i}\left(L_{i}, V_{i}\right)} \quad \text { for } i=\mathrm{VM}, \mathrm{VL}, \mathrm{RF}
$$

where $F_{E M G, i}$ is the EMG corrected force for muscle $i$ at length $L_{i}$ and velocity $V_{i}, F_{M V C, i}$ is the $\mathrm{MVC}$ force and $A M P N_{i}$ is the normalised EMG amplitude.
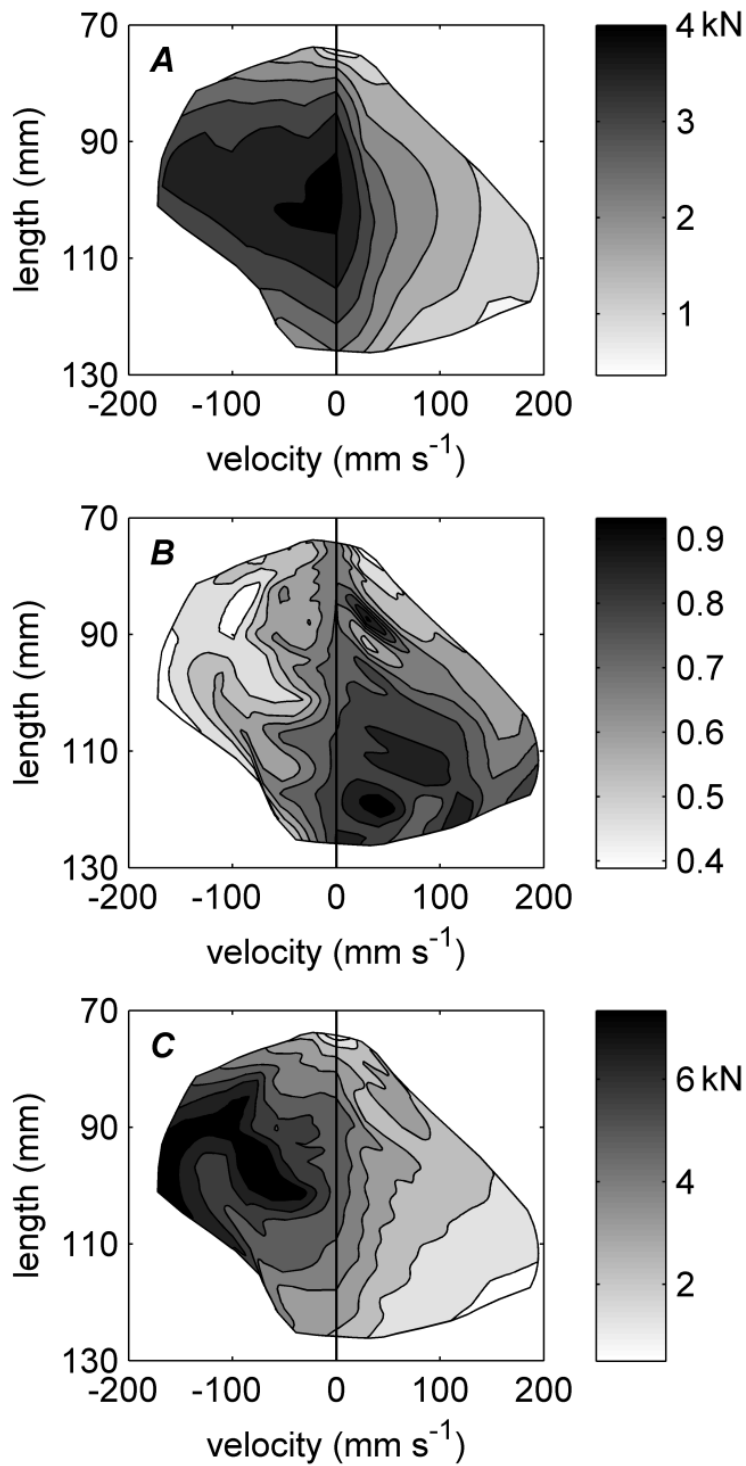

Figure 1. Contour graphs detailing the data generated for each muscle group and subject from the isovelocity dynamometer and EMG measurements. These comprised: MVC force - length - velocity surface $(A)$; normalised EMG amplitude - length - velocity surface $(B)$; and EMG corrected force - length velocity surface $(C)$. The final graph was obtained by dividing the MVC force surface by the normalised EMG amplitude surface.

\section{In vitro tetanic force function}

The in vitro tetanic force - velocity relationship was approximated by a classical Hill-type hyperbola in the concentric region and a rectangular hyperbola in the eccentric region (Yeadon et al., 2006). This function is defined by four parameters: maximum eccentric force 
$\left(F_{\text {ecc }}\right)$; maximum isometric force $\left(F_{o}\right)$; maximum shortening velocity $\left(V_{\max }\right)$ and the curvature of the concentric hyperbola $\left(V_{d} / V_{\max }\right)$. It was fitted to both the MVC and EMG corrected peak force - velocity data on a subject specific bases. The measured isometric forces were excluded from this process as they were used to help assess the goodness of fit of the tetanic function.

Table 1. Upper and lower bounds used in the optimisations for each of the four parameters defining the tetanic force - velocity function

\begin{tabular}{ccl}
\hline parameter & bounds & reference \\
\hline$F_{\text {ecc }}$ & $\left(F_{\text {ecd }} / F_{o}\right)=1.3-1.7$ & Dudley et al. (1990) \\
$F_{o}$ & $F_{o, \text { measured }} \pm 50 \%$ & From the measurements \\
& $400-1400 \mathrm{~mm} \cdot \mathrm{s}^{-1}$ & $\sim 4.4-12.7$ optimal fibre lengths per second \\
$V_{\max }$ & (Spector et al., 1980; Fitts et al., 1989) \\
$V_{c}$ & $\left(V_{c} / V_{\max }\right)=0.15-0.5$ & Pertuzon \& Bouisset (1972), Faulkner et al. (1986) \\
\hline
\end{tabular}

\section{Joint basis}

A simpler alternative to the individual muscle modelling was also assessed. The individual muscle EMG amplitudes were combined to approximate a knee extensors amplitude $\left(A M P N_{K E}\right)$, which was then be used to correct $\left(T_{E M G}\right)$ the measured MVC joint torques $\left(T_{M V C}\right)$ :

$$
T_{E M G} \cong \frac{T_{M V C}}{A M P N_{K E}} \quad \text { where } A M P N_{K E}=\sum_{V M, V L, R F}\left(\frac{P C S A_{i}}{P C S A_{\text {total }}} A M P N_{i}\right)
$$

This approach has the advantage of not requiring a muscle model; physiological crosssectional area (PCSA) of the knee extensor muscles is the only additional piece of information required (Chow et al 1999).

\section{Statistical analysis}

The goodness of fit of the tetanic force function to the measured MVC and EMG corrected forces was assessed based on the following values, providing information on the goodness of fit of the in vitro tetanic function to the measured forces (I and III), and the tetanic nature of the fit (II and IV).

I. Normalised RMS difference score between the tetanic and measured forces:

$$
\left(\frac{1}{F_{\text {ecc }}} \sqrt{\frac{1}{n} \sum_{i=1}^{n}\left|F_{\text {tetanic }}-F_{\text {measured }}\right|^{2}}\right)
$$

II. Ratio of eccentric to isometric tetanic force parameters: $\left(F_{e c c} / F_{o}\right)$

III. Difference between tetanic and measured isometric forces: $\left(100 \% \times\left(F_{\text {o,measured }}-F_{\text {o,tetanic }}\right) / F_{\text {o,measured }}\right)$

IV. Tetanic shortening velocity parameters: $\left(V_{c} / V_{\max }\right)$ and $V_{\max }$

V. Ratio of tetanic eccentric forces between the MVC and EMG corrected data: $\left(F_{\text {ecc, EMG }} / F_{\text {ecc, MVC }}\right)$ 
The first four of these measures were assessed using paired samples t-tests $(p \leq 0.05)$ (SPSS version 13.0; SPSS Inc., Chicago, IL, USA).

\section{RESULTS}

Typical results for the MVC forces, normalised EMG amplitude and EMG corrected forces are shown in Fig. 2. The results from fitting the in vitro tetanic force - velocity function to the MVC and EMG corrected force measurements are presented in Tables $2-3$ and Fig. 3. Table 2 gives the fitted and measured isometric forces, where the fitted values were obtained independently of the measurements. Table 3 gives the goodness of fit comparison measures and those showing a statistically significant difference between MVC and EMG corrected forces are highlighted. Examples of the in vitro tetanic force - velocity function fit to MVC and EMG corrected peak forces for each muscle group are presented in Fig. 3. Retests showed no noticeable loss of performance throughout the trials (all within $3-8 \%$ of the original torque values).

Table 2. Measured and fitted isometric forces for the MVC and EMG corrected data

\begin{tabular}{|c|c|c|c|c|c|}
\hline \multirow{2}{*}{$\begin{array}{l}\text { muscle } \\
\text { group }\end{array}$} & \multirow[b]{2}{*}{ subject } & \multicolumn{2}{|c|}{$\mathrm{MVC}$ isometric forces } & \multicolumn{2}{|c|}{ EMG corrected isometric forces } \\
\hline & & $\begin{array}{l}\text { measured } \\
(\mathrm{N})\end{array}$ & $\begin{array}{l}\text { fitted } \\
(\mathrm{N})\end{array}$ & $\begin{array}{c}\text { measured } \\
(\mathrm{N})\end{array}$ & $\begin{array}{l}\text { fitted } \\
(\mathrm{N})\end{array}$ \\
\hline \multirow{5}{*}{ VM } & S1 & 2176 & 1836 & 3410 & 3211 \\
\hline & S2 & 1616 & 1366 & 2286 & 2044 \\
\hline & S3 & 1693 & 1443 & 2076 & 1980 \\
\hline & S4 & 1420 & 1309 & 2444 & 2102 \\
\hline & S5 & 1792 & 1533 & 2091 & 2811 \\
\hline \multirow{5}{*}{ VL } & S1 & 4130 & 3489 & 5197 & 5145 \\
\hline & S2 & 3066 & 2591 & 4463 & 3716 \\
\hline & S3 & 3212 & 2736 & 3761 & 3804 \\
\hline & S4 & 2694 & 2484 & 3770 & 3614 \\
\hline & S5 & 3400 & 2910 & 5227 & 5669 \\
\hline \multirow{5}{*}{$\mathrm{RF}$} & S1 & 1480 & 1256 & 2035 & 1824 \\
\hline & S2 & 1099 & 928 & 1623 & 1495 \\
\hline & S3 & 1202 & 981 & 1336 & 1262 \\
\hline & S4 & 966 & 891 & 1389 & 1356 \\
\hline & S5 & 1219 & 1042 & 1772 & 1689 \\
\hline
\end{tabular}



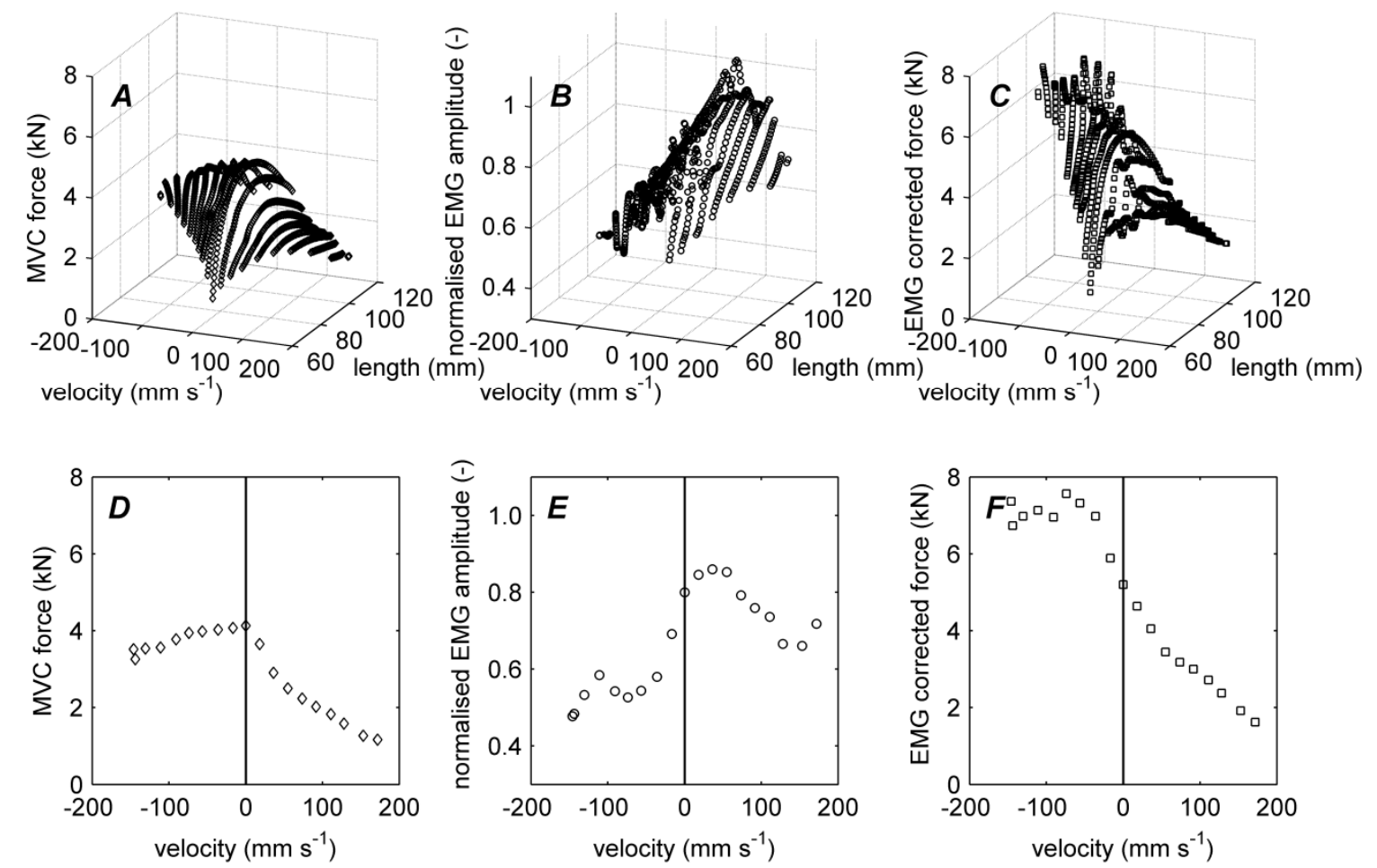

Figure 2. MVC force $(A \& D)$, normalised EMG amplitude $(B \& E)$, and EMG corrected force $(C \& F)$ measurements. Data is presented as a function of fibre length and velocity $(A-C)$ and along the velocity line of peak force $(D-F)$. Illustrated for the VL of subject S1.
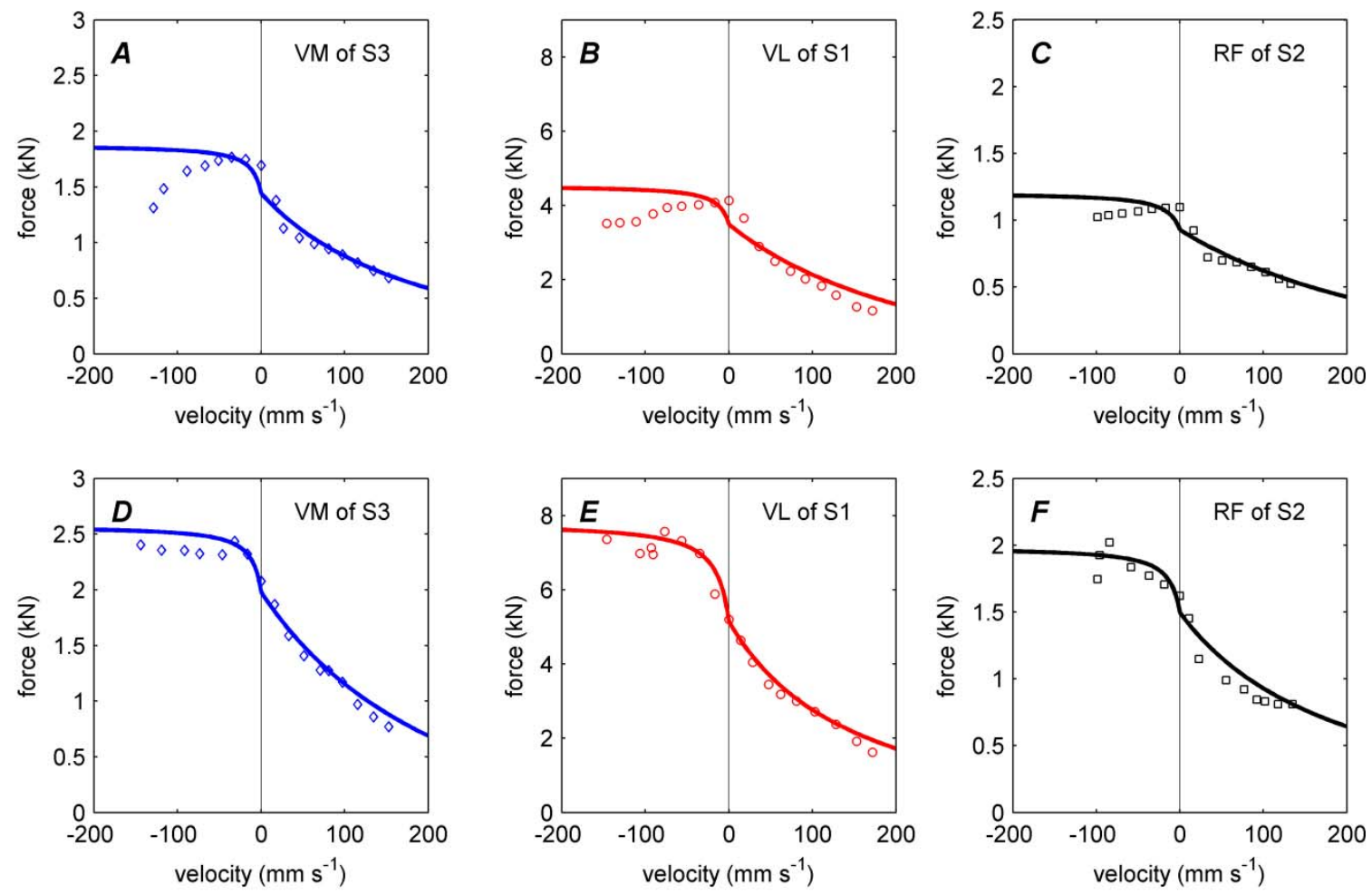

Figure 3. Tetanic force - velocity fits to the MVC $(A-C)$ and EMG corrected $(D-F)$ forces. Illustrative results for: VM of subject S3 ( $A \& D)$; VL of subject $\mathrm{S} 1(B \& E)$; and RF of subject S2 $(C \& F)$. The solid lines represent the optimised tetanic curve fits and the open symbols represent the measured forces. 
RMS difference between the in vitro tetanic fit and measured forces and percent difference between the in vitro tetanic fit and measured $F_{o}$ values were both significantly lower for EMG corrected forces compared to $\mathrm{MVC}$ forces. The ratio $F_{e c c} / F_{o}$ was significantly higher for EMG corrected forces compared to MVC forces, and for the latter hit the lower bound of 1.30 for all five subjects. There were no significant differences in shortening velocity parameters $V_{c} / V_{\max }$ and $V_{\max }$. The ratio of fitted maximum eccentric force, $F_{\text {ecc,EMG }} / F_{\text {ecc,MVC, }}$ had an overall value of $1.58 \pm 0.19$.

Typical results for the in vitro tetanic function fitted to the MVC and EMG corrected joint torques are given in Fig. 4, where the EMG corrected torques were obtained using eq.(1). The more accurate approach of summing torques from the individual muscles to gain EMG corrected joint torque gave very similar results to those presented in Fig. 4: RMS differences of $6-18 \mathrm{Nm}$ compared to $13-19 \mathrm{Nm}$; and isometric torque difference of $-12-6 \%$ compared to $-10-8 \%$. This supports the simplified approach, given by eq.(1), for estimating EMG corrected joint torque.
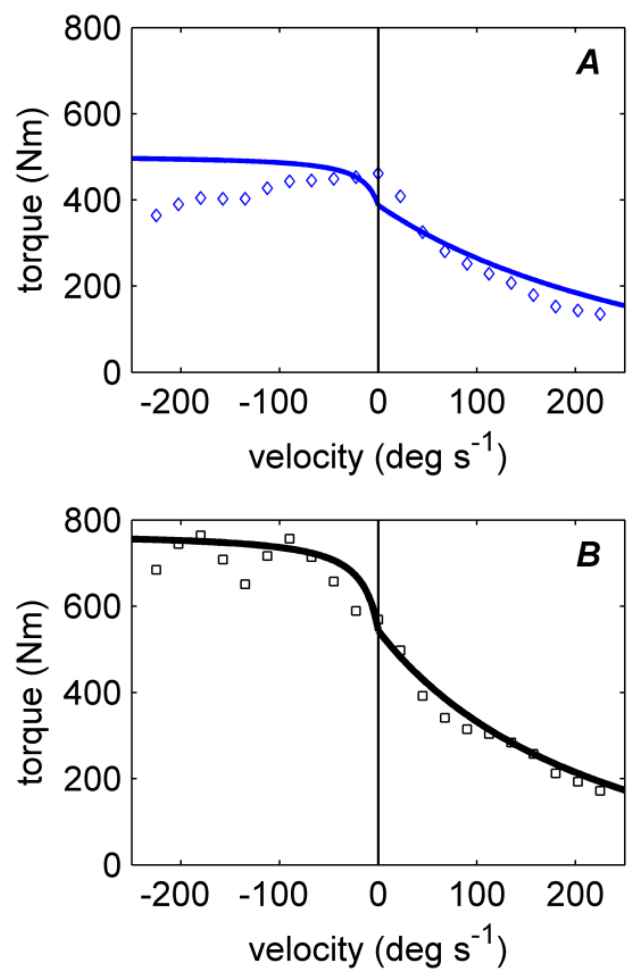

Figure 4. Joint torque - angular velocity for the MVC $(A)$ and EMG corrected $(B)$ torques. EMG corrected torques were obtained simply by combining EMG amplitudes according to eq.(1). The solid lines represent the optimised tetanic curve fits and the open symbols represent the measured torques. Illustrated for subject S1.

\section{DISCUSSION}

Visual inspection of the graphs in Fig. 3 suggests that the EMG corrected forces are closer to a classical in vitro tetanic force - velocity curve compared to the MVC forces. The main improvement is the raising of eccentric forces to a plateau nearly 1.4 times higher than isometric, a ratio similar to that obtained using transcutaneous electrical stimulation of the knee extensors (Dudley et al., 1990; Westing et al., 1990).

Statistically the EMG corrected forces were a significantly better approximation to the in vitro tetanic profile than the MVC forces (Table 3). This was not strongly reflected in the normalised RMS score because of increased random noise in the EMG corrected force measurements. EMG signals, especially those from a single pair of electrodes, have greater 
variance than dynamometer measurements (Staudenmann et al., 2006) and introduce this variability to the EMG corrected forces. Also, the fits to the MVC forces already achieved a relatively low RMS difference, typically $7-9 \%$, as there were four free parameters to optimise and concentric forces should not be strongly affected by changes in neural factors. However, every MVC force optimisation hit the lower bound for the ratio $F_{\text {ecc }} / F_{o}$ which was not the case for the EMG corrected optimisations.

The third comparison measure is based on the difference between the in vitro tetanic fit and measured maximum isometric force, $F_{o}$. For the MVC data the in vitro tetanic fit $F_{o}$ was consistently less than the measured $F_{o}$, while for the EMG corrected data the in vitro tetanic fit $F_{o}$ could fall either side of the measured $F_{o}$ and the two were significantly closer (Tables 2 and 3). In order for the in vitro tetanic fit to the MVC data to obtain a low RMS error, $F_{o}$ had to be systematically underestimated to account for the low MVC eccentric forces (illustrated in Fig. 3).

The ratio $V_{c} / V_{\max }$ describes the degree of curvature in the concentric force - velocity hyperbola. Changes in neural factors are not expected to have a marked effect on concentric forces and based simply on this premise neural factors may not be expected to strongly affect either $V_{c} / V_{\max }$ or maximum shortening velocity, $V_{\max }$. However, the majority of the fits to the MVC data hit the upper bound for $V_{c} / V_{\max }$, due to the tetanic fit $F_{o}$ being dragged to its lower bound to account for the low MVC eccentric forces (Fig. 3 and Table 3). The fits to the EMG corrected data had more freedom in selecting the degree of curvature in the concentric force - velocity hyperbola since the in vitro tetanic fit $F_{o}$ values were more realistic.

The fitting of the in vitro tetanic function to the force measurements allowed all four of the force - velocity parameters to vary within quite large but (tetanically) realistic bounds (Table 1) and assessed how well the resulting curves matched the established in vitro tetanic profile. Despite the freedom in this fitting approach, every MVC curve hit the lower bound for $F_{\text {ecc }} / F_{o}$ and $75 \%$ hit the upper bound for $V_{c} / V_{\max }$. In comparison, less than half of the EMG corrected curves hit a bound, suggesting that this data provides a better and realistic approximation to the classical in vitro tetanic force - velocity profile.

The closeness of the EMG corrected forces to the classical in vitro tetanic force - velocity profile supports neural factors as the primary contributor to the difference between MVC and in vitro tetanic forces, although contributions from other factors cannot be ruled out, e.g. non-linear changes in pennation angle (Rutherford \& Jones, 1992; Herbert \& Gandevia, 1995; Aagaard et al., 2001) or myofacial force transmission (Rijkelijkhuizen et al., 2005). Using reported data for changes in quadriceps pennation angle under load (Fukunaga et al., 1997), gives a reduction in tendon force of up to 3\% when the knee extensors are put under maximal isometric tension. This would account for most of the difference between the mean EMG corrected $F_{\text {ecc }} / F_{o}$ value determined in this study $(1.34 \pm 0.06)$ and the value of 1.4 reported for experiments using purely sub maximal subcutaneous electrical stimulation (Dudley et al., 1990). Care is required with such a comparison since changes in pennation angle are implicitly included in the measured value for $F_{e c c} / F_{o}$ in the latter study. However, the level of stimulation was well below maximal and so pennation angle changes would have had a smaller effect on measured torque compared to this study. A value of 1.31 for $F_{\text {ecc }} / F_{o}$ has been reported based purely on maximal tolerable stimulation, whilst using both MVC and stimulation resulted in higher absolute torques but a lower value for $F_{e c c} / F_{o}$ of 1.23 (Westing et al., 1990). Thus, it appears that not only is the ratio $F_{e c c} / F_{o}$ limited to well below theoretical levels but that as $F_{\text {ecc }}$ increases in absolute terms, the value $F_{\text {ecc }} / F_{o}$ tends to drop even with the aid of electrical stimulation. It is hypothesized that neural factors are present to act against excessively overloading the musculoskeletal system. To safely increase eccentric strength by decreasing the level of neural factors concomitant increases in resistance to 
loading of the tendons, bones, and other structural tissues would be necessary. This implies that marked changes in neural factors are only likely to be seen over long periods of training, longer than those normally assessed in training studies.

The process of generating MVC and EMG corrected forces from MVC torques required a number of assumptions. The derivation of individual muscle forces assumed negligible cocontraction. Normalised EMG amplitudes of the hamstrings were no higher than 0.08 around peak torque for all contraction velocities, which is not sufficiently high to have affected the results presented. Also, EMG amplitude was normalised to a measured maximum assuming that: this was the true maximum of the EMG signal in the sense that it represented all fibres being maximally activated; and this maximum was the same over the length - velocity phase space of the knee extension task (Figs $1 B$ and $2 B$ ). Despite these assumptions RMS errors in the optimised fits were low, suggesting that although these areas require further work they are unlikely to have represented major limitations.

The results presented suggest that EMG amplitude can be used to estimate tetanic forces from maximal in vivo force measurements, and neural factors are a major contributor to the difference between in vitro and in vivo maximal force. If eccentric strength depression is largely due to an involuntary change in neural factors, it may be questioned whether it is present in more natural movements, and if it can be manipulated or mitigated?

\section{REFERENCES}

Aagaard, P., Simonsen, E.B., Andersen, J.L., Magnusson, S.P., Halkjaer-Kristensen, J., Dyhre-Poulsen, P., 2000. Neural inhibition during maximal eccentric and concentric quadriceps contraction: effects of resistance training. Journal of Applied Physiology 89, 2249-2257.

Aagaard, P., Andersen, J.L., Dyhre-Poulsen, P., Leffers, A.M., Wagner, A., Magnusson, S.P., Halkjaer-Kristensen, J., Simonsen, E.B., 2001. A mechanism for increased contractile strength of human pennate muscle in response to strength training: changes in muscle architecture. Journal of Physiology 534, 613-623.

Beltman, J. G. M., Sargeant, A. J., van Mechelen, W., de Haan, A., 2004. Voluntary activation level and muscle fiber recruitment of human quadriceps during lengthening contractions. Journal of Applied Physiology 97(2): 619-626.

Chow, J.W., Darling, W.G., Ehrhardt, J.C., 1999. Determining the force-length-velocity relations of the quadriceps muscles: II. Maximum muscle stress. Journal of Applied Biomechanics, 15, 191-199.

Dudley, G.A., Harris, R.T., Duvoisin, M.R., Hather, B.M., Buchanan, P., 1990. Effect of voluntary vs. artificial activation on the relationship of muscle torque to speed. Journal of Applied Physiology 69, 2215-2221.

Edman, K.A.P., Elzinga, G., Noble, M.I.M., 1978. Enhancement of mechanical performance by stretch during tetanic contractions of vertebrate skeletal muscle fibres. Journal of Physiology 281, 139-155.

Faulkner, J.A., Clafin, D.R., McCully, K.K., 1986. Power output of fast and slow fibers from human skeletal muscles. In: Jones, N.L., McCartney, N., McComas, A.J. (Eds), Human Muscle Power. Human Kinetics, Champaign, pp. 81-94.

Fitts, R.H., Costill, D.L., Gardetto, P.R., 1989. Effect of swim exercise on human muscle fiber function. Journal of Applied Physiology 66, $465-475$.

Forrester, S.E., Pain, M.T.G., 2007. Maximising the resolution of EMG characteristics from dynamic contractions by combining a muscle model and wavelet analysis. In 
Proceedings of the 31st Annual Meeting of the American Society of Biomechanics. University of Stanford, USA.

Fukunaga, T., Ichinose, Y., Ito, M., Kawakami, Y., Fukashiro, S., 1997. Determination of fascicle length and pennation in a contracting human muscle in vivo. Journal of Applied Physiology 82, 354-358.

Harry, J.D., Ward, A.W., Heglund, N.C., Morgan, D.L., McMahon, T.A., 1990. Cross-bridge cycling theories cannot explain high-speed lengthening behaviour in frog muscle. Biophysical Journal 57, 201-208.

Hawkins D, Hull ML., 1990. A method for determining lower extremity muscle-tendon lengths during flexion/extension movements. Journal of Biomechanics 23, 487-494.

Herbert, R.D., Gandevia, S.C., 1995. Changes in pennation with joint angle and muscle torque: in vivo measurements in human brachialis muscle. Journal of Physiology 484, $523-532$

Hill, A.V., 1938. The heat of shortening and the dynamic constants of muscle. Proceedings of the Royal Society of London. Series B 126, 135-142.

Hoy, M.G., Zajac, F.E., Gordon, M.E., 1990. A musculoskeletal model of the human lower extremity: The effect of muscle, tendon, and moment arm on the moment-angle relationship of musculotendon actuators at the hip, knee, and ankle. Journal of Biomechanics 23, 157-169.

Jacobs, R., Bobbert, M.F., Van Ingen Schenau, G.J., 1996. Mechanical output from individual muscles during explosive leg extensions: The role of biarticular muscles. Journal of Biomechanics 29, 513-523.

Karamanidis, K., Arampatzis, A., 2006. Mechanical and morphological properties of human quadriceps femoris and triceps surae muscle-tendon unit in relation to aging and running. Journal of Biomechanics 39, 406-417.

Karlsson, J.S., Yu, J. \& Akay, M., 2000. Time-frequency analysis of myoelectric signals during dynamic contractions: A comparative study. IEEE Transactions on Biomedical Engineering 47, 228-238.

Kellis, E., Baltzopoulos, V., 1999. In vivo determination of the patella tendon and hamstrings moment arms in adult males using videofluoroscopy during submaximal knee extension and flexion. Clinical Biomechanics 14, 118-124.

Pertuzon, E., Bouisset, S., 1972. Biomechanics of monoarticulated movement and mechanical properties of muscle. Le Travail humain 35, 364.

Pierrynowski, M.R., Morrison, J.B., 1985. A physiological model for the evaluation of muscular forces in human locomotion: Theoretical aspects. Mathematical Biosciences 75, 69-101.

Rijkelijkhuizen, J.M., Baan, G.C., de Haan, A., de Ruiter, C.J., Huijing, P.A., 2005. Extramuscular myofascial force transmission for in situ rat medial gastrocnemius and plantaris muscles in progressive stages of dissection. Journal of Experimental Biology 208, 129-140.

Rutherford, O.M., Jones, D.A., 1992. Measurement of fibre pennation using ultrasound in the human quadriceps in vivo. European Journal of Applied Physiology 65, 433-437.

Seger, J.Y., Thorstensson, A., 1994. Muscle strength and myoelectric activity in prepubertal and adult males and females. European Journal of Applied Physiology and Occupational Physiology 69, 81-87. 
Spector, S.A., Gardiner, P.F., Zernicke, R.F., Roy, R.R., Edgerton, V.R., 1980. Muscle architecture and force-velocity characteristics of cat soleus and medial gastrocnemius: implications for motor control. Journal of Neurophysiology 44, 951-960.

Staudenmann, D., Kingma, I., Daffertshofer, A., Stegeman, D.F., van Dieën, J.H., 2006. Improving emg-based muscle force estimation by using a high-density emg grid and principal component analysis. IEEE Transactions on Bio-medical Engineering 53, 712719.

von Tscharner, V., 2000. Intensity analysis in time-frequency space of surface myoelectric signals by wavelets of specified resolution. Journal of Electromyography and Kinesiology 10, 433-445.

Westing, S.H., Cresswell, A.G., Thorstensson, A., 1991. Muscle activation during maximal voluntary eccentric and concentric knee extension. European Journal of Applied Physiology and Occupational Physiology 62, 104-108.

Westing, S.H., Seger, J.Y., Thorstensson, A., 1990. Effects of electrical stimulation on eccentric and concentric torque-velocity relationships during knee extension in man. Acta physiologica Scandinavica 140, 17-22.

Yeadon, M.R., King, M.A., Wilson, C., 2006. Modelling the maximum voluntary joint torque/angular velocity relationship in human movement. Journal of Biomechanics 39, $476-482$. 


\section{APPENDIX A. KNEE EXTENSIONS MUSCLE MODEL}

The individual muscle forces were determined by assuming that the measured joint torque $\left(T_{J}\right)$ was entirely due to the actions of the knee extensors:

$$
T_{J}=\sum_{i=\mathrm{RF}, \mathrm{VM}, \mathrm{VL}, \mathrm{VI}} F_{T, i} \times d_{M, i}
$$

where $F_{T}$ is the tendon force and $d_{M}$ the moment arm (Kellis \& Baltzopoulos, 1999). Hence, assuming that muscle force is directly proportional to physiological cross-sectional area (PCSA), and neglecting differences in pennation angle, the fibre force $\left(F_{F}\right)$ was estimated from:

$$
F_{F} \cos \alpha=F_{T}=\frac{T_{J}}{d_{M}} f_{P C S A}
$$

where $f_{P C S A}$ is the fractional PCSA and $\alpha$ the pennation angle (determined assuming a constant muscle width; Pierrynowski \& Morrison, 1985).

The fibre length $\left(L_{F}\right)$ was determined from:

$$
L_{F}=\frac{L_{M T}-L_{T}}{\cos \alpha}
$$

The muscle-tendon length $\left(L_{M T}\right)$ was obtained from:

$$
L_{M T}=L_{M T}^{R E F}+\int_{\theta}^{\theta^{R E F}} d_{M}(\theta) \mathrm{d} \theta
$$

where $L_{M T}^{R E F}$ is the muscle-tendon reference length (Hawkins and Hull, 1990).

The tendon length $\left(L_{T}\right)$ was obtained from:

$$
F_{T}=k_{T}\left(L_{T}-L_{T S L}\right)
$$

where $k_{T}$ is the tendon stiffness, determined based on a tendon strain of $6 \%$ at maximum isometric force (Karamanidis \& Arampatzis, 2006). The corresponding velocities were obtained by differentiating the length data. All muscle parameters were obtained from the literature and then scaled to the subjects of this study (Table A.1). 
Table A1. Muscle model parameters.

(a) Literature values

\begin{tabular}{|c|c|c|c|c|c|}
\hline & VM & VL & VI & $\mathrm{RF}$ & Notes \\
\hline $\begin{array}{c}\text { Optimal fibre length, } \\
L_{F O}(\mathrm{~mm})\end{array}$ & 93 & 93 & 93 & 81 & \multirow{3}{*}{$\begin{array}{l}\text { body mass, } 77.8 \mathrm{~kg} \\
\text { leg length, } 0.898 \mathrm{~m} \\
\text { (Jacobs et al., 1996) }\end{array}$} \\
\hline $\begin{array}{l}{ }^{1} \text { Maximum isometric } \\
\text { force, } F_{F O}^{M A X}(\mathrm{~N})\end{array}$ & \multicolumn{3}{|c|}{5400 (total for the vastii) } & 930 & \\
\hline $\begin{array}{c}{ }^{1} \text { Tendon slack length, } \\
L_{T S L}(\mathrm{~mm})\end{array}$ & 160 & 160 & 160 & 360 & \\
\hline $\begin{array}{c}{ }^{2} \text { Pennation angle at } \\
L_{F O}, \alpha_{P O}\left(^{\circ}\right)\end{array}$ & 4.5 & 4.5 & 4.5 & 5 & (Hoy et al., 1990) \\
\hline $\begin{array}{c}{ }^{3} \mathrm{PCSA}\left(\mathrm{mm}^{2}\right) \\
\left(f_{P C S A}\right)\end{array}$ & $\begin{array}{c}4405 \\
(0.216)\end{array}$ & $\begin{array}{c}8358 \\
(0.410)\end{array}$ & $\begin{array}{c}4637 \\
(0.227)\end{array}$ & $\begin{array}{r}2994 \\
(0.147)\end{array}$ & (Chow et al., 1999) \\
\hline
\end{tabular}

(b) Subject values (mean \pm SD)

\begin{tabular}{cccc}
\hline & VM & VL & RF \\
\hline Optimal fibre length, $L_{F O}(\mathrm{~mm})$ & $88 \pm 4$ & $88 \pm 4$ & $1135 \pm 152$ \\
\hline Maximum isometric force, $F_{F O}^{M A X}(\mathrm{~N})$ & $1669 \pm 223$ & $3167 \pm 423$ & $357 \pm 18$ \\
\hline Tendon slack length, $L_{T S L}(\mathrm{~mm})$ & $152 \pm 9$ & $184 \pm 11$ & 5 \\
\hline Pennation angle at $L_{F O}, \alpha_{P O}\left(^{\circ}\right)$ & 4.5 & 4.5 & $53 \pm 5$ \\
\hline Tendon stiffness, $k_{T}\left(\mathrm{kN} \mathrm{m}^{-1}\right)$ & $183 \pm 18$ & $286 \pm 28$ & \\
\hline
\end{tabular}

Muscle properties were scaled to the individual subjects using the ratios:

(i) SR1 (for forces) = ratio of body masses;

(ii) SR2 (for lengths) = ratio of leg lengths; and

(iii) $\mathrm{SR} 3$ (for moment arms) $=\sqrt{S R 1 / S R 2}$ 
Hawkins, D., Hull, M.L., 1990. A method for determining lower extremity muscle-tendon lengths during flexion/extension movements. Journal of Biomechanics 23, 487-494.

Hoy, M.G., Zajac, F.E., Gordon, M.E., 1990. A musculoskeletal model of the human lower extremity: the effect of muscle, tendon, and moment arm on the moment-angle relationship of musculotendon actuators at the hip, knee, and ankle. Journal of Biomechanics 23, 157-169.

Jacobs, R., Bobbert, M.F., Van Ingen Schenau, G.J., 1996. Mechanical output from individual muscles during explosive leg extensions: the role of biarticular muscles. Journal of Biomechanics 29, 513-523.

Karamanidis, K., Arampatzis, A., 2006. Mechanical and morphological properties of human quadriceps femoris and triceps surae muscle-tendon unit in relation to aging and running. Journal of Biomechanics 39, 406-417.

Kellis, E., Baltzopoulos, V., 1999. In vivo determination of the patella tendon and hamstrings moment arms in adult males using videofluoroscopy during submaximal knee extension and flexion. Clinical Biomechanics 14, 118-124.

Pierrynowski, M.R., Morrison, J.B., 1985. A physiological model for the evaluation of muscular forces in human locomotion: theoretical aspects. Mathematical Biosciences 75, 69-101. 